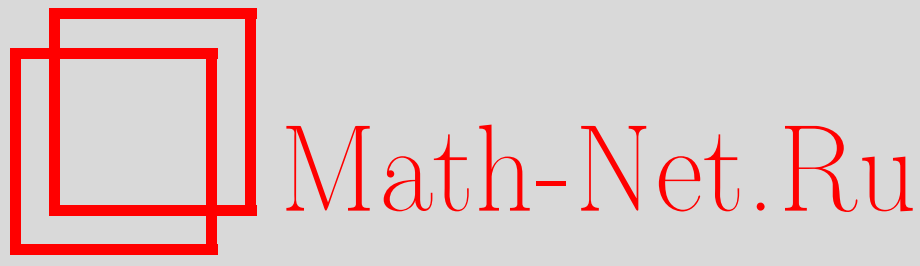

Обшероссийский математический портал

О. Б. Пламеневская, Типичный гомеоморфизм не обладает свойством липшицева отслеживания, Матем. заметки, 1999, том 65, выпуск 3, 477-480

DOI: https://doi.org/10.4213/mzm1074

Использование Общероссийского математического портала Math-Net.Ru подразумевает, что вы прочитали и согласны с пользовательским соглашением http://www.mathnet.ru/rus/agreement

Параметры загрузки:

IP : 35.173 .219 .149 
26 апреля 2023 г., 14:20:30

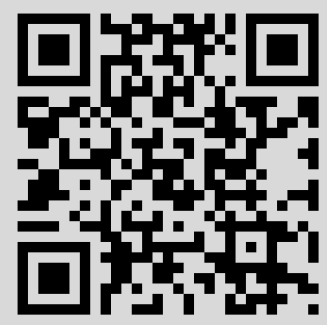




\section{ТИПИЧНЫЙ ГОМЕОМОРФИЗМ НЕ ОБЛАДАЕТ СВОЙСТВОМ ЛИПШИЦЕВА ОТСЛЕЖКИВАНИЯ}

\section{О.Б. Пламеневская}

Пусть $M$ - гладкое замкнутое многообразие с римановой метрикой dist.

Рассмотрим пространство $Z(M)$ дискретных динамических систем (гомеоморфизмов) на $M$ с $C^{0}$-топологией, индуцированной метрикой

$$
\rho_{0}(\varphi, \psi)=\max _{x \in M}\left\{\operatorname{dist}(\varphi(x), \psi(x)), \operatorname{dist}\left(\varphi^{-1}(x), \psi^{-1}(x)\right)\right\} .
$$

Пусть $\delta>0$. Последовательность $\left\{x_{k}\right\}_{k \in \mathbb{Z}}$ назьвается $\delta$-траекторией системы $\varphi$, если

$$
\operatorname{dist}\left(\varphi\left(x_{k}\right), x_{k+1}\right)<\varepsilon, \quad k \in \mathbb{Z} .
$$

Задача отслеживания псевдотраекторий заключается в нахождении настоящих траекторий данной системы, близких (в каком-либо смысле) к ее $\delta$-траекториям. Сформулируем несколько определений (см. [1]).

Система $\varphi$ обладает свойством отслежсивания, если при любом $\varepsilon>0$ существует $\delta>0$ такое, что для всякой $\delta$-траектории $\left\{x_{k}\right\}$ найдется точка $x \in M$, для которой

$$
\operatorname{dist}\left(\varphi^{k}(x), x_{k}\right)<\varepsilon, \quad k \in \mathbb{Z} .
$$

Система $\varphi$ обладает свойством липиичева отслеживания (с константами $L, d>0$ ), если при любом $\delta \in(0, d)$ для всякой $\delta$-траектории $\left\{x_{k}\right\}$ найдется точка $x \in M$, для которой

$$
\operatorname{dist}\left(\varphi^{k}(x), x_{k}\right)<L \delta, \quad k \in \mathbb{Z} .
$$

Пусть $B_{\varepsilon}(A)$ обозначает $\varepsilon$-окрестность множества $A \subset M$.

Система $\varphi$ обладает свойством слабого отслеживания, если при любом $\varepsilon>0$ сушествует $\delta>0$ такое, что для всякой $\delta$-траектории $\left\{x_{k}\right\}$ найдется траектория $O(x)$ некоторой точки $x \in M$, для которой

$$
\left\{x_{k}\right\} \subset B_{\varepsilon}(O(x)) \text {. }
$$

Свойство элементов топологического пространства $X$ называется типичным, если оно выполняется для всех элементов некоторого массивного подмножества $Y \subset X ; Y$ массивно, если оно содержит счетное пересечение открытых всюду плотных подмножеств $X$.

Пространство $X$ назьвается пространством Бәра, если любое его массивное подмножество всюду плотно. Классическая теорема Бэра утверждает, что всякое полное метрическое пространство является пространством Бэра. Несложно видеть, что $Z(M)$ - полное пространство.

(C) О.Б. ПлАмЕНЕвСКАя 
Типичность свойства слабого отслеживания в пространстве $Z(M)$ доказана $\mathrm{P}$. Корлессом и С. Ю. Пилюгиным [2]. Типичность свойства отслеживания в случае $\operatorname{dim} M \leqslant 3$ доказана К. Одани [3]. Доказательство типичности отслеживания для произвольной размерности фазового пространства $M$ получено в [4].

Цель данной заметки - показать, что в отличие от свойства отслеживания свойство липшицева отслеживания не является типичным в $Z(M)$. Более того, типичный гомеоморфизм не обладает свойством липшицева отслеживания. Мы определяем также свойство липшицева слабого отслеживания и показываем, что типичный гомеоморфизм не обладает даже этим более слабым свойством.

Система $\varphi$ обладает свойством липшищева слабого отслеживания (с константами $L, d>0$ ), если при любом $\delta \in(0, d)$ для всякой $\delta$-траектории $\left\{x_{k}\right\}$ найдется траектория $O(x)$, для которой

$$
\left\{x_{k}\right\} \subset B_{L \delta}(O(x)) .
$$

Выделим сначала некоторый класс гомеоморфизмов многообразия $M$, не имеющих липшицева отслеживания с заданными константами. Для фиксированной точки $p \in M$ будем обозначать

$$
B_{R}=\{x \in M: \operatorname{dist}(x, p) \leqslant R\}, \quad S_{R_{1}, R_{2}}=\left\{x \in M: R_{2} \leqslant \operatorname{dist}(x, p) \leqslant R_{1}\right\} .
$$

Лемма. Пусть гомеоморфизм $\varphi$ имеет неподвижную точку р. Предположим, что существуют числа $R_{1}, \ldots, R_{5}$,

$$
R_{1}>R_{2}>R_{3}>R_{4}>R_{5}>0
$$

dתs которых

$$
\begin{gathered}
\varphi\left(S_{R_{1}, R_{3}}\right) \subset \operatorname{int} S_{R_{2}, R_{3}}, \\
\varphi\left(B_{R_{4}}\right) \subset \operatorname{int} B_{R_{5}},
\end{gathered}
$$

причем выполняются неравенства

$$
\begin{gathered}
R_{2}-R_{4}<d, \\
R_{1}-R_{3}>2 L\left(R_{2}-R_{4}\right), \\
R_{3}-R_{5}>2 L\left(R_{2}-R_{4}\right) .
\end{gathered}
$$

Тогда гомеоморфизм $\varphi$ не имеет липшичева отслеживания с константами $L$ и $d$.

ДоКАЗАТЕЛЬСТВо. Положим

$$
\delta=R_{2}-R_{4}
$$

Построим $\delta$-траекторию системы $\varphi$ следующим образом. Выберем точку $y$ так, что

$$
\operatorname{dist}(y, p)=\frac{R_{1}+R_{3}}{2} .
$$

Тогда $y \in S_{R_{1}, R_{3}}$ и в силу (2) будет $\operatorname{dist}(\varphi(y), p)<R_{2}$. Найдем точку $z$ такую, что

$$
\operatorname{dist}(\varphi(y), z)<\delta, \quad \operatorname{dist}(z, p)<R_{4} .
$$

Последовательность

$$
\ldots, \varphi^{-1}(y), y, \varphi(y), z, \varphi(z), \varphi^{2}(z), \ldots
$$

является $\delta$-траекторией гомеоморфизма $\varphi$. Предположим, что она отслеживается с точностью $L \delta$ траекторией некоторой точки $x \in M$. Можно считать, что $\operatorname{dist}(x, y)<L \delta$. Из неравенства (5) и выбора $\delta$ следует, что $L \delta$-окрестность точки $y$ целиком лежит в $S_{R_{1}, R_{3}}$, откуда $x \in S_{R_{1}, R_{3}}$. Тогда

$$
\varphi^{k}(x) \in \operatorname{int} S_{R_{2}, R_{3}}, \quad k \geqslant 1,
$$

поскольку включение (2) влечет положительную инвариантность множества int $S_{R_{2}}, R_{3}$. В то же время $z \in B_{R_{4}}$, значит $\varphi(z) \in B_{R_{5}}$ в силу (3). По предположению об отслеживании должно быть

$$
\operatorname{dist}\left(\varphi(z), \varphi^{3}(x)\right)<L \delta,
$$

однако вместе с (9) это противоречит неравенству (6). Таким образом, построенная $\delta$-траектория не отслеживается с точностью $L \delta$, что доказьвает лемму, так как $\delta<d$ по неравенству (4). 
Теорема 1. Типичный гомеоморфизм не обладает свойством липшичева отслехивания.

ДокАЗАТЕльство. Введем подмножество $G(L, d)$ пространства $Z(M)$, считая $\varphi \in G(L, d)$, если для некоторого натурального $n$ гомеоморфизм $\varphi^{n}$ удовлетворяет условиям леммы.

Очевидно, что при любых $L, d>0$ множество $G(L, d)$ открыто. Докажем, что оно плотно в $Z(M)$.

Предположим сначала, что некоторый гомеоморфизм $\varphi$ имеет неподвижную точку $p$. Покажем, что в любой окрестности $\varphi$ найдется гомеоморфизм, удовлетворяющий условиям леммы.

Выберем достаточно малое $R>0$ и будем изменять $\varphi$ на множестве $B_{R}(p)$. Забиксируем числа $R_{1}, \ldots, R_{5}$ такие, что $R>R_{1}$ и выполняются неравенства (1) и (4)-(6). Пользуясь тем, что $p-$ неподвижная точка $\varphi$, найдем $r_{1}$ такое, что

$$
\varphi\left(B_{r_{1}}(p)\right) \subset \operatorname{int} B_{R_{2}}(p) .
$$

Считая $r_{1}<R_{1}$, найдем отображение, “сжимающее" шар $B_{R_{1}}(p)$ в $B_{r_{1}}(p)$, т.е. построим гомеоморфизм $\alpha: M \rightarrow M$ такой, что

$$
\left.\alpha\right|_{M \backslash B_{R}(p)}=\mathrm{id}, \quad \alpha(p)=p, \quad \alpha\left(B_{R_{1}}(p)\right)=B_{r_{1}}(p) .
$$

Заменив при необходимости $\varphi$ гомеоморфизмом $\varphi \circ \alpha$ (который мы снова будем обозначать через $\varphi$ ), добьемся справедливости включения

$$
\varphi\left(B_{R_{1}}(p)\right) \subset \operatorname{int} B_{R_{2}}(p) .
$$

Далее, $\varphi\left(S_{R_{1}, R_{3}}\right) \not \supset p$, так как $\varphi(p)=p$, а тогда в силу компактности множества $S_{R_{1}, R_{3}}$ для некоторого $r_{3}>0$ будет

$$
\varphi\left(S_{R_{1}, R_{3}}\right) \subset \operatorname{int} S_{R_{2}, r_{3}} .
$$

Если $r_{3} \geqslant R_{3}$, то условие (2) выполняется. В противном случае построим гомеоморфизм $\beta: M \rightarrow M$ со свойствами

$$
\left.\beta\right|_{M \backslash B_{R_{2}}(p)}=\mathrm{id}, \quad \beta(p)=p, \quad \beta\left(B_{r_{3}}(p)\right)=B_{R_{3}}(p) .
$$

Отображение $\beta$ переводит $S_{R_{2}, r_{3}}$ в $S_{R_{2}, R_{3}}$, поэтому для гомеоморфизма $\beta$ о $\varphi$ верно включение (2).

Действуя аналогично, еще раз поменяем гомеоморфизм внутри шара $B_{R_{3}}(p)$ так, чтобы выполнялось включение (3). Полученный гомеоморфизм удовлетворяет условиям леммы и отличается от исходного лишь внутри $B_{R}(p)$. Если $R$ достаточно мало, расстояние между новьм и старым гомеоморфизмами также будет мало.

Итак, мы показали, что в любой окрестности гомеоморфизма, имеющего неподвижную точку, найдется гомеоморфизм из множества $G(L, d)$. Теперь найдем гомеоморфизм из $G(L, d)$ в произвольном открытом множестве $V \subset Z(M)$. В $V$ найдется гомеоморфизм $\varphi$, у которого есть периодические точки. Это вытекает, например, из следующего факта: если $x$ - неблуждающая точка системы $\varphi$, то существуют последовательности $x_{k} \in M$ и $\varphi_{k} \in Z(M)$, для которых $\varphi_{k} \rightarrow \varphi$, $x_{k} \rightarrow x$, причем $x_{k}$ - периодическая точка $\varphi_{k}$ (см. [1, теорема 1.4.1]). Пусть $\varphi^{n}(p)=p$. Изменяя $\varphi$ в малых окрестностях точек $p$ и $\varphi^{n-1}(p)=\varphi^{-1}(p)$ аналогично случаю неподвижной точки, можно построить гомеоморфизм $\psi \in V, n$-я степень которого удовлетворяет условиям леммы.

Итак, при любых $L, d>0$ множество $G(L, d)$ открыто и всюду плотно. Зафиксируем последовательности $L_{n} \rightarrow \infty$ и $d_{n} \rightarrow 0$ и рассмотрим множество

$$
G=\bigcap_{n \geqslant 0} G\left(L_{n}, d_{n}\right) .
$$

Очевидно, $G$ - массивное множество. Покажем, что гомеоморфизмы из множества $G$ не обладают свойством липшицева отслеживания. Предположим, что это не так, т.е. некоторый гомеоморфизм $\varphi \in G$ имеет липшицево отслеживание с константами $L$ и $d$. Тогда любая степень $\varphi$ имеет липшицево отслеживание с теми же константами (это легко проверяется непосредственно). Поэтому $\varphi \notin G\left(L_{n}, d_{n}\right)$ при $L_{n}>L, d_{n}<d$, а это противоречит включению $\varphi \in G$. Теорема доказана. 
ТЕОРема 2. Типичный гомеоморфизм в $Z(M)$ не обладает свойством липиицева слабого отслеживания.

ДоКАЗАТЕЛЬСтво. Несложно видеть, что гомеоморфизмы, описанные в лемме, не обладают свойством липшицева слабого отслеживания с константами $L$ и $d$. Доказательство теоремы 1 не годится для случая слабого отслеживания лишь потому, что свойство слабого отслеживания для $\varphi$ не влечет наличия этого свойства у $\varphi^{n}$. Мы покажем, однако, что если $\Phi=\varphi^{n}$ обладает описанными в лемме свойствами, причем для некоторых $R^{\prime}>R_{1}$ и $R^{\prime \prime} \in\left(R_{3}, R_{4}\right)$ выполнено включение

$$
\Phi^{-1}\left(S_{R^{\prime}, R^{\prime \prime}}\right) \subset \operatorname{int} S_{R^{\prime}, R^{\prime \prime}}
$$

то $\varphi$ не имеет липшицева слабого отслеживания. Добавленное условие аналогично условиям леммы, поэтому рассуждение из теоремы 1 будет доказьвать и теорему 2.

Предположим, что гомеоморфизм $\Phi=\varphi^{n}$ удовлетворяет условиям леммы, а также условию (10). Выберем настолько малое $R>0$, что

$$
\bigcup_{0<k<n} \varphi^{k}\left(B_{R}(p)\right) \cap B_{R}(p)=\varnothing .
$$

Будем считать, что $R>R^{\prime}$. Как и раньше, определим $\delta$ равенством (7). Построим для системы $\varphi$ $\delta$-траекторию вида

$$
\ldots, \varphi^{-1}(y), y, \varphi(y), \ldots, \varphi^{n-1}(y), z, \varphi(z), \varphi^{2}(z), \ldots,
$$

по-прежнему выбрав точку $y$ так, чтобы выполнялось (8), а $z \in B_{R_{4}}(p)$ так, чтобы было верно неравенство $\operatorname{dist}\left(\varphi^{n-1}(y), z\right)<\delta$.

Допустим, что построенная $\delta$-траектория слабо отслеживается с точностью $L \delta$ некоторой траекторией $O(x)$ системы $\varphi$. Тогда в $L \delta$-окрестности точки $y$, которая по неравенству (4) и выбору $y(8)$ целиком лежит в $S_{R_{1}, R_{3}}$, найдется хотя бы одна точка траектории $O(x)$. Можно считать, что $x \in S_{R_{1}, R_{3}}$. Мы придем к противоречию, показав, что

$$
\operatorname{dist}\left(O(x), \varphi^{n}(z)\right)>L \delta .
$$

Поскольку (2) верно для $\Phi$, при положительных $k$ кратных $n$ выполняется включение $\varphi^{k}(x) \in$ $S_{R_{2}, R_{3}}$. Из неравенства (5) следует, что

$$
\operatorname{dist}\left(\varphi^{k}(x), \varphi^{n}(z)\right)>L \delta,
$$

так как $\varphi^{n}(z) \in B_{R_{5}}(p)$. Если же $k$ не делится на $n$, то неравенство (12) сразу вытекает из (11).

При отрицательных $k$ точки $\varphi^{k}(x)$ также не могут лежать в $L \delta$-окрестности точки $\varphi^{n}(z)$. Если $k$ не делится на $n$, это следует из (11), а при отрицательных $k$ кратных $n$ имеем $\varphi^{k}(x) \in S_{R^{\prime}, R^{\prime \prime}}$ в силу (10). Теорема доказана.

Я благодарю своего научного руководителя С. Ю. Пилюгина за постановку задачи.

\section{СПИСОК ЦИТИРОВАННОЙ ЛИТЕРАТУРЫ}

1. Pilyugin S. Yu. The Space of Dynamical Systems with the $C^{0}$-Topology. Lecture Notes in Math. V. 1571. Berlin-Heidelberg: Springer, 1994. 2. Corless R., Pilyugin S. Yu. // J. Math. Anal. Appl. 1995. V. 189. P. 409-423. 3. Odani K. // Proc. Amer. Math. Soc. 1990. V. 110. P. 281-284. 4. Pilyugin S. Yu., Plamenevskaya O. B. Shadowing is generic // Topology Appl. (to appear). 